\title{
A FIXED POINT THEOREM FOR IMAGE-INTERSECTING MAPPINGS
}

\author{
ALEXANDER ABIAN
}

\begin{abstract}
Many of the known fixed point theorems of order reversing mappings of partially or simply ordered sets into themselves pertain to dense such ordered sets. In this paper a fixed point theorem is given for an order reversing mapping from a not necessarily dense simply ordered set into itself.
\end{abstract}

Let $x$ and $y$ be elements of a simply ordered set $(S,<)$. By the "open at $x$ and closed at $y$ interval" of $S$, we mean, as usual, $(x, y]$ or $[y, x)$ depending on whether $x \leqslant y$ or $y \leqslant x$.

Based on the above, we prove:

THEOREM. Let $(S, \leqslant)$ be a nonempty simply ordered set in which every nonempty bounded above subset has a least upper bound. Let $f$ be a mapping from $S$ into $S$ such that $f$ is order reversing, i.e.,

$$
x \leqslant y \text { implies } f(x) \geqslant f(y) \text { for every } x, y \in S
$$

and such that every nonempty open at $x$ and closed at $f(x)$ interval $H$ of $S$ has a nonempty intersection with its image $F[H]$, i.e.,

$$
\begin{aligned}
& \text { for every } x \in S \text { if } x \neq f(x) \text { then } \\
& f[H] \cap H \neq \varnothing \text { where } H=(x, f(x)] \text { or } H=[f(x), x) .
\end{aligned}
$$

Then $f$ has a fixed point.

Proof. Since $S$ is nonempty, $a \in S$ for some $a$. If $a \leqslant f(a)$ then by (1) we have $f(a) \geqslant f(f(a))$, and if $a \geqslant f(a)$ then, again by (1) we have $f(a)<$ $f(f(a))$. Thus, there exist elements $p$ and $q$ of $S$ such that:

$$
p \leqslant q \text { with } p \leqslant f(p) \text { and } q=f(p)
$$

(indeed, if $a \leqslant f(a)$ we take $p=a$ and $q=f(a)$, and, if $a>f(a)$ we take $p=f(a)$ and $q=f(f(a)))$.

From (3) it follows that the subset $E$ of $S$ given by:

$$
E=\{x \mid p \leqslant x \leqslant q \text { and } x \leqslant f(x)\}
$$

Received by the editors April 18, 1978 and, in revised form, June 2, 1978.

AMS (MOS) subject classifications (1970). Primary 06A05, 47H10.

Key words and phrases. Fixed point, simply ordered set. 
is nonempty and bounded above. Let

$$
e=\operatorname{lub} E \text {. }
$$

Clearly,

$$
p<e .
$$

We claim that $e$ is a fixed point of $f$, i.e.,

$$
e=f(e) \text {. }
$$

Let us assume on the contrary. Thus, one of the two cases below must occur:

FIRST CASE. $e<f(e)$. But then from (6) by (1) and (3) it follows:

$$
p \leqslant e<f(e)<q .
$$

Since $e<f(e)$, by (2) there exists $m \in S$ such that

$$
e<m \leqslant f(e) \text { and } e<f(m) \leqslant f(e)
$$

which by (8) implies:

$$
p \leqslant m \leqslant q \text { and } p \leqslant f(m) \leqslant q .
$$

However, if $m \leqslant f(m)$ then by (10) and (4) we would have $m \in E$ which by (5) would imply $m \leqslant e$, contradicting (9). On the other hand, if $m>f(m)$ then by (1) we would have $f(m) \leqslant f(f(m))$ which by (10) and (4) would imply $f(m) \in E$, which, in turn, by (5) would imply $f(m)<e$, again contradicting (9).

Hence, the first case cannot occur.

SECOND CASE. $e>f(e)$. Hence, again by (2) there exists $m \in S$ such that:

$$
f(e) \leqslant m<e \text { and } f(e) \leqslant f(m)<e .
$$

However, since $e>f(e)$ and since by (11) we have $m<e$ and $f(m)<e$, from (4) and (5) it would then follow that there exists $h \in S$ such that:

$$
m<h \text { and } f(m)<h \leqslant f(h)
$$

contradicting (1).

Hence, the second case also cannot occur.

Thus, our assumption is false and (7) is established.

REMARK 1. The following example shows that condition (2) by itself does not imply the existence of a fixed point.

Let $f$ map the real closed interval $[0,3]$ into itself (where reals are taken in their usual order) such that $f(x)=2.5$ for $0 \leqslant x \leqslant 1$ and $f(x)=0.5$ for $1<x \leqslant 2$ and $f(x)=1.5$ for $2<x \leqslant 3$.

It can be readily verified that $f$ satisfies (2) and yet $f$ has no fixed point.

REMARK 2. As mentioned earlier, it is not required that $(S, \leqslant)$ be dense. Indeed, let $S=\{a, b\}$ with $a<b$ and $f(a)=f(b)=b$. Clearly, the hypotheses of the Theorem are satisfied and yet $S$ is not dense.

REMARK 3. The statement of the Theorem remains valid if in it "nonempty open at $x$ and closed at $f(x)$ interval" is replaced by "nonempty open at $f(x)$ 
and closed at $x$ interval" and the proof remains unchanged.

REMARK 4. The statement of the Theorem does not remain valid if in it "nonempty open at $x$ and closed at $f(x)$ interval" is replaced by "nonempty open at $x$ and open at $f(x)$ interval". For instance, let $S=\{a, b\}$ with $a<b$ and let $f(a)=b$ and $f(b)=a$. Then (since there are no nonempty open intervals in $S$ ) every nonempty open interval of $S$ has a nonempty intersection with its image under $f$. However, $f$ has no fixed point.

Similarly, the same example shows that the statement of the Theorem does not remain valid if in it "nonempty open at $x$ and closed at $f(x)$ interval" is replaced by "nonempty closed at $x$ and closed at $f(x)$ interval".

For related ideas see[1] to [8].

The author thanks the referee for valuable suggestions.

\section{REFERENCES}

1. A. Abian and A. Brown, A theorem on partially ordered sets, with applications to fixed point theorems, Canad. J. Math. 13 (1961), 78-82.

2. A. Abian, A fixed point theorem for nonincreasing mappings, Boll. Un. Mat. Ital. 2 (1969), 200-201.

3. H. Hoeft and M. Hoeft, Some fixed point theorems for partially ordered sets, Canad. J. Math. 28 (1976), 992-997.

4. F. T. Metcalf and T. H. Payne, On the existence of fixed points in a totally ordered set, Proc. Amer. Math. Soc. 31 (1972), 441-444.

5. T. B. Muenzenberger and R. E. Smithson, Fixed point theorems for acyclic and dendritic spaces, Pacific J. Math. 72 (1977), 501-512.

6. Z. Shmuely, Fixed points of antitone mappings, Proc. Amer. Math. Soc. 52 (1975), 503-505.

7. R. E. Smithson, Fixed points of order preserving multifunctions, Proc. Amer. Math. Soc. 28 (1971), 304-310.

8. , Fixed points in partially ordered sets, Pacific J. Math. 45 (1973), 363-367.

Department of Mathematics, Iowa State University, Ames, Iowa 50011 